\title{
(3+1) assembly of three human telomeric repeats into an asymmetric dimeric G-quadruplex
}

\author{
Na Zhang, Anh Tuân Phan* and Dinshaw J. Patel \\ Structural Biology Program, Memorial Sloan-Kettering Cancer Center \\ New York, NY 10021, USA
}

Supporting Information

*Corresponding author: phantuan@mskcc.org

Running title: $(3+1)$ G-quadruplex assembly in the human telomere

Key words: $(3+1)$ G-quadruplex assembly; asymmetric dimeric G-quadruplex; telomeric DNA 


\section{SUPPORTING TABLES}

Table S1. List of analog-substituted sequences used for resonance assignments. 8-BrG (and 8-BrA) substitutions of $\mathrm{G}$ (and $\mathrm{A}$ ) resulted in disappearance of peaks for corresponding $\mathrm{H} 8$ protons. T-to-U substitutions resulted in disappearance of peaks for methyl protons and appearance of peaks for H5.

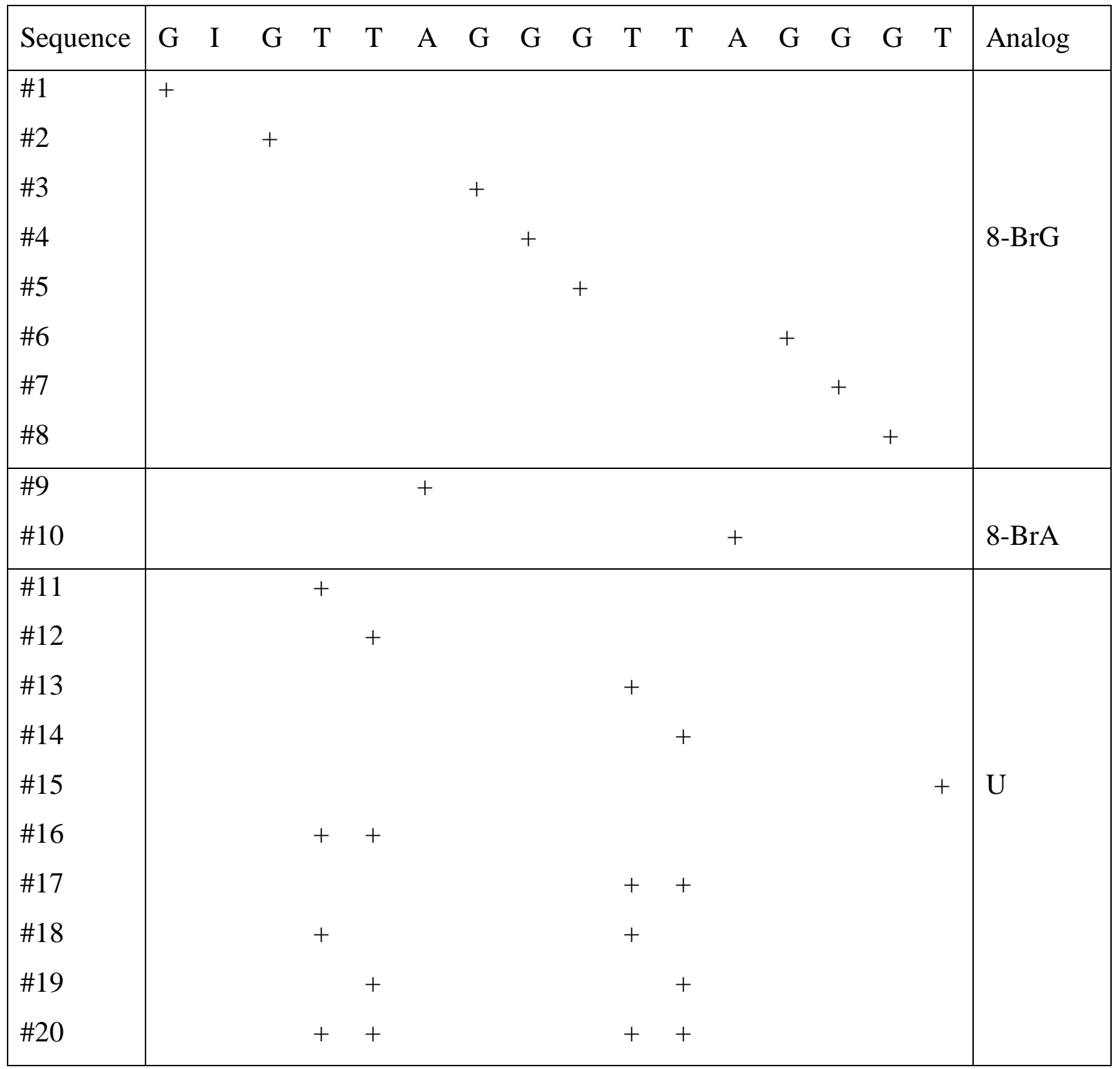


Table S2. List of three-repeat and single-repeat human telomeric DNA sequences used for strand mixing experiments. In all cases, NMR spectra indicated formation of a heterodimeric G-quadruplex association of two strands.

\begin{tabular}{|c|c|}
\hline Three-repeat sequences & Single-repeat sequences \\
\hline GIGTTAGGGTTAGGGT & TAGGGU \\
GIGTTAGGGTTAGGGT & TAGGGT \\
GIGTTAGGGTTAGGGT & AGGGT \\
GIGTTAGGGTUAGGGT & TTAGGGT \\
GIGTTAGGGTTAGGGU & TAGGGT \\
GGGTTAGGGTTAGGGT & AGGGT \\
GGGTTAGGGTTAGGGTTA & AGGGT \\
AGGGTTAGGGTTAGGGTT & TAGGGT \\
TAGGGTTAGGGTTAGGGT & TAGGGT \\
\hline
\end{tabular}




\section{SUPPORTING FIGURES}

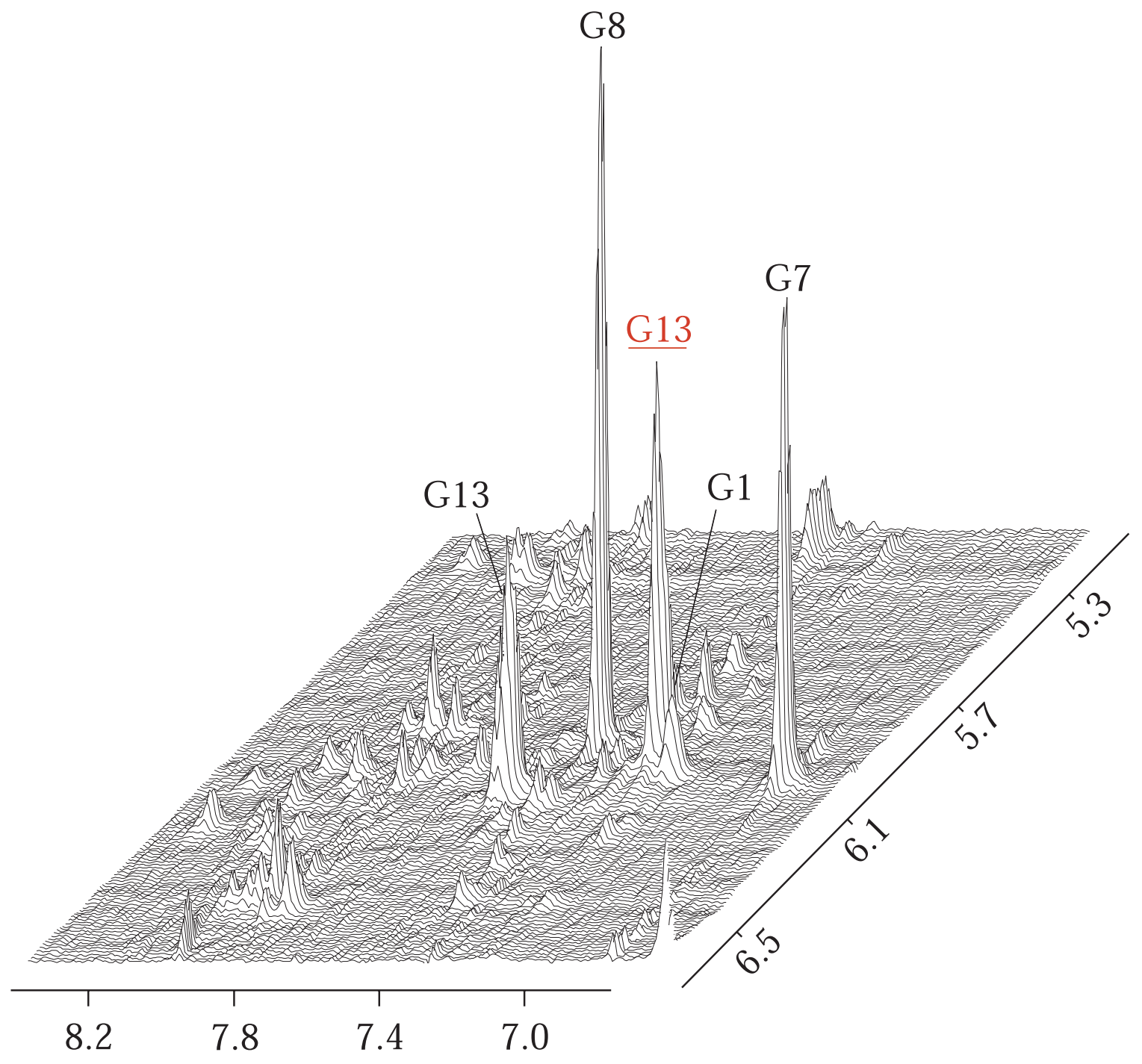

Figure S1. Stacked plot of the NOESY spectrum (mixing time, $50 \mathrm{~ms}$ ) of htel3I showing five strong intra-residue $\mathrm{H} 8-\mathrm{H} 1$ ' cross-peaks for syn guanines. 
(a)
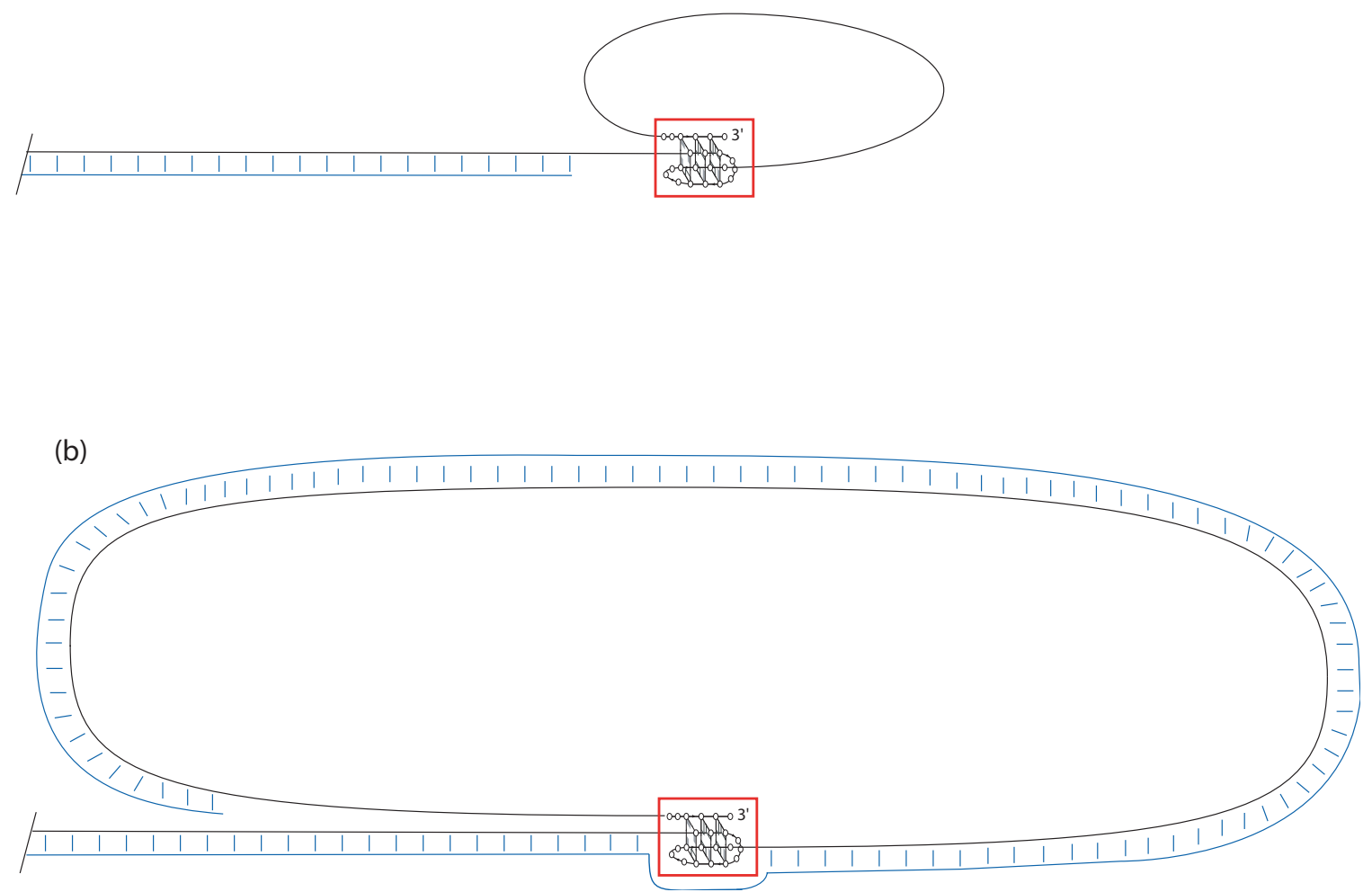

Figure S2. Possible models of $(3+1)$ G-quadruplexes in the human telomere, within the 3'-end overhang (a) and within the junction of the t-loop (b). Guanine-rich strand is shown in black; cytosine-rich strand is blue; the (3+1) G-quadruplex is highlighted in a red box. 